Preface: II Russian-Spanish Congress on Particle and Nuclear Physics at All Scales, Astroparticle Physics and Cosmology

Alexander Andrianov, Domènec Espriu, Vladimir Andrianov, and Sergei Kolevatov

Citation: AIP Conference Proceedings 1606, 1 (2014);

View online: https://doi.org/10.1063/1.4891110

View Table of Contents: http://aip.scitation.org/toc/apc/1606/1

Published by the American Institute of Physics 


\section{PREFACE: II Russian-Spanish Congress on Particle and Nuclear Physics at All Scales, Astroparticle Physics and Cosmology}

This publication contains the proceedings of the II Russian-Spanish Congress on Particle and Nuclear Physics at All Scales, Astroparticle Physics and Cosmology, a collection of refereed papers presented in plenary and parallel sessions at a meeting that gathered leading Russian and Spanish Scientists in the above fields in Saint-Petersburg from October $1^{\text {st }}$ through October $4^{\text {th }}$ 2013 (http://hep.phys.spbu.ru/conf/esp-rus2013/).

The meeting was organized and supported by Saint-Petersburg State University with the purpose of strengthening already existing collaborations and initiate new ones involving scientists from Russia and Spain. More than 100 Russian and Spanish participants joined for this Congress. The first Congress of this series was held in the Fall of 2011 in Barcelona

(http://icc.ub.edu/congress/ESP-RUS2011/). Participants in that first meeting concluded that the existing Spanish-Russian collaborations, some of them going back to many years ago, were quite successful but in order to reinforce them and extend their scope it would be convenient to continue this kind of Congresses in future.

On this second edition many topics were covered in the Congress. An incomplete list includes: modern gravity and cosmology, dark energy, theoretical implications of extra space dimensions, deviations from Newton dynamics, dark matter and axions, search for physics beyond the Standard Model at ultra-high energies both on colliders and in space, strong interactions of hadrons at high energies accessible at the Large Hadron Collider (LHC), relativistic heavy ion physics at the LHC and RHIC and predictions of new phases in hot and dense nuclear matter, physics of neutron-rich nuclei, search for new isotopes (ISOLDE), AdS/CFT and AdS/QCD correspondence in quantum field theory and string theory and holographic models of strong interactions, quantization of gauge theories, exact results in SUSY theories, Casimir effect, complex systems and self-organization, etc. More details as well as the slides of the different talks can be found on the web-page http://hep.phys.spbu.ru/conf/esp-rus2013/program.html.

The organizers and participants of the II Russian-Spanish Congress are very grateful for support to the following institutions: Committee of External Relations of the Saint Petersburg Government, Saint Petersburg Scientific Center of the Russian Academy of Sciences, Non-profit Dmitry Zimin Foundation “Dynasty”, and Russian Foundation for Basic Research.

A third edition of this series of meetings is planned for 2015 in Santiago de Compostela, Spain.

The Editors: $\quad$ Alexander Andrianov, chairman Domènec Espriu, co-chairman Vladimir Andrianov, co-chairman Sergei Kolevatov

II Russian-Spanish Congress on Particle and Nuclear Physics at all Scales, Astroparticle Physics and Cosmology AIP Conf. Proc. 1606, 1-2 (2014); doi: 10.1063/1.4891110 (C) 2014 AIP Publishing LLC 978-0-7354-1242-2/\$30.00 